\title{
STRATEGIE DI TRADUZIONE DELLA METAFORA ALLA LUCE DELLA LINGUISTICA COGNITIVA
}

\begin{abstract}
Bocian Edyta, Strategie di traduzione della metafora alla luce della linguistica cognitiva [Translation strategy of metaphor in the hight of cognitive linguistics]. Studia Romanica Posnaniensia, Adam Mickiewicz University Press, Poznań, vol. XXXVI: 2009, pp. 15-32. ISBN 978-83-232-2035-0. ISSN 0137-2475.
\end{abstract}

The article consists of two parts connected with each other logically. In the first one the author presents the basics of the cognitive theory of metaphor by Lakoff and Johnson. Whereas in the second, basing on the theses above, she perfoms an analysis of translation strategies of metaphor on the basis of the contemporary Italian prose (Baricco, Ammaniti). The translation processes described include such phenomena as: word for word translation, demethaphoring, cancellation of metaphor and literary translation.

1. Le riflessioni che seguono trovano origine nella teoria cognitivista ${ }^{1}$ della metafora, elaborata soprattutto da Lakoff, professore di Linguistica e Scienza Cognitiva, e da Johnson, professore di Filosofia del linguaggio, di cui il cardine si poggia senz'altro sulla constatazione che la metafora è onnipresente nel linguaggio umano, il che significa che essa non è una proprietà esclusiva della prosa e in particolare della poesia, ma appare in percentuale molto elevata anche nella lingua quotidiana. Secondo Lakoff e Johnson (1998a: 21) la metafora è infatti presente sia nel linguaggio letterario che in quello comune, contrariamente a quanto si è abituati a pensare:

La metafora è da molti considerata come uno strumento dell'immaginazione poetica, un artificio retorico, qualcosa insomma che ha piü a che vedere con il linguaggio straordinario che con quello comune. Non solo, la metafora è anche tipicamente considerata, come caratteristica del solo livello linguistico, una questione di parole piuttosto che di pensiero

' La teoria cognitivista della metafora viene presentata in questa sede in base agli scritti: Lakoff, Johnson (1998a, 1998b, 2002), Lakoff (1991), Jäkel (2003), Gaeta, Nuraghi (2003), Dirven, Verspoor (1999), Krzeszowski (2005), Rollo (2004). 
o di azione. Per questa ragione molti pensano di poter fare benissimo a meno della metafora. Noi abbiamo invece trovato che la metafora è diffusa ovunque nel linguaggio quotidiano, e non solo nel linguaggio ma anche nel pensiero e nell'azione: il nostro comune sistema concettuale, in base al quale pensiamo e agiamo, è essenzialmente di natura metaforica.

Difatti, anche se è difficile crederci, tutti noi viviamo nel mondo della cosiddetta metafora onnipresente ${ }^{2}$ in quanto tutti i giorni, in maniera più o meno cosciente, ricorriamo alla metafora nelle conversazioni quotidiane. Ovviamente la tesi sulla metaforicità del linguaggio non è per niente nuova, essa ha trovato riscontro in vari altri approcci sulla metafora, sin dai tempi remoti, già Aristotele nella Retorica, come ci fa notare Giovanni Manetti, parla del suo carattere universale in quanto procedimento stilistico di uso generale ${ }^{3}$. Però a questo punto la scoperta tra virgolette di Lakoff e Johnson è apparsa ai suoi contemporanei come una trovata brillante in quanto, come si sa benissimo, il più difficile da vedere è quello che si ha sotto gli occhi $^{4}$.

Alla luce di quanto detto prima non stupisce affatto il vivo interesse dalla parte di diversi studiosi rivolto appunto alla nozione della metafora. Su questo tema sono stati infatti versati fiumi d"inchiostro lasciandosi dietro un vero oceano di scritti ${ }^{5}$.

${ }^{2}$ Mi riferisco qui al titolo del libro $W$ świecie powszechnej metafory che Jerzy Świątek ha dedicato alla metafora, puntando infatti sul suo carattere universale.

${ }^{3}$ Cfr. Manetti 2005: 29-34.

${ }^{4}$ La stessa osservazione, la esprime anche Jäkel (2003: 20) nelle seguenti parole: «w dostrzeżeniu tego powszechnego zjawiska prawdopodobnie przeszkadza jego pospolitość oraz towarzyszący mu efekt przyzwyczajenia ».

${ }^{5}$ In primo luogo, nel corso dei secoli, gli studiosi hanno cercato di risolvere il problema dell'appartenenza della metafora, dando vita a tre correnti diverse di pensiero. La prima sostiene che la metafora è una deviazione semantica per il fatto che invece di impiegare la designazione socialmente consacrata per un oggetto o un concetto, il parlante adopera una designazione impropria. La seconda vede nella metafora una forma costitutiva del linguaggio, per cui la metafora non è una deviazione semantica ma è il linguaggio ad essere organicamente metaforico. La terza invece crede che la metafora sia una forma costitutiva del pensiero per il fatto che non è il linguaggio ad essere organicamente metaforico, bensì il nostro sistema concettuale per mezzo del quale noi agiamo e pensiamo.

In secondo luogo, gli studiosi si sono posti come scopo di spiegare il meccanismo della metafora, ossia come essa funzioni. In linea di massima, secondo Olaf Jäkel (2003: 95), possiamo distinguere tre principali approcci alla metafora in quanto le teorie della metafora ruotano intorno ad essi. « Niemal wszystkie przyczynki do współczesnej dyskusji poświęconej metaforze można z łatwością zaliczyć do jednego „obozu”, charakteryzowanego przez wybrane tu określenia kierunku » (Jäkel, 2003: 95). Il primo approccio viene chiamato la teoria di sostituzione e di comparazione, il secondo la teoria interattiva e il terzo la teoria pragmatica di reinterpretazione. La teoria di sostituzione e di comparazione nasce con Aristotele e Quintiliano, essa sostiene che la metafora equivale alla sostituzione di una parola per mezzo di un'altra secondo la definizione « la metafora è l'applicazione di un nome estraneo all'oggetto, trasferito o dal genere alla specie, o dalla specie al genere o da specie a specie o per analogia 》 (Manetti, 2005: 46) basandosi sul meccanismo della somiglianza. Il secondo approccio, ideato da Richards e sviluppato da Black, vede nella metafora una specie di interazione tra i significati delle parole usate nella creazione dell'immagine metaforica. Il terzo approccio, infine, di cui il padre è Searle, considera che la 
Va da sé dunque, visto che la metafora fa parte della nostra vita quotidiana, e non solo della letteratura, e dato che su di essa si sono aperte tante discussioni, che il problema della sua traduzione dovrebbe essere fondamentale, per non dire il più discusso, nell' ambito della teoria della traduzione, specialmente in chiave linguistica. Tuttavia i fatti dimostrano il contrario ${ }^{6}$.

Daniela Pirazzini (1997: 9) spiega questo disinteresse elencando, a ragione, due cause che secondo lei sono alla base dello scarso interesse per la traduzione delle metafore dalla parte della Übersetzungswissenschaft, ma secondo noi valgono anche per altre scuole di traduzione. La prima è che le metafore sono un fenomeno tipico dei testi di qualità letteraria e, di conseguenza, materia di competenza della critica letteraria e della filosofia, e la seconda che esse non pongono problemi diversi dalla traduzione in genere.

2. Questo articolo si pone dunque lo scopo di presentare le difficoltà che incontrano i traduttori di fronte alle metafore per mezzo dell'illustrazione delle strategie di traduzione applicate. Gli esempi che seguiranno provengono dall'analisi del corpus linguistico composto da 6 opere di letteratura contemporanea italiana. Quattro delle sei sono nate sotto la penna di Alessandro Baricco, e le altre due sotto quella di Niccolò Ammaniti, due autori la cui prosa abbonda di immagini metaforiche. Le opere in questione sono, per quanto riguarda Baricco: Seta, City, Oceano mare, Castelli di rabbia, e per quanto concerne Ammaniti: Ti prendo e ti porto via, Fango. Le traduttrici sono tre ${ }^{7}$ : Halina Kralowa (Seta, Oceano mare, Castelli di rabbia), Joanna Ugniewska (City) e Dorata Duszyńska (Ti prendo e ti porto via, Fango). Nell'analisi delle strategie di traduzione proponiamo di servirci degli strumenti d'indagine proposti dalla teoria cognitivista della metafora quali: il dominio di partenza e di arrivo e la mappatura.

metafora non é un fenomeno semantico bensì pragmatico in quanto la metafora rimanda ad un significato diverso da quello letterale.

${ }^{6}$ Tabakowska sostiene apertamente quanto segue: « Metafora nigdy nie znajdowała się w centrum zainteresowania teorii przekładu o orientacji językoznawczej, choć na jej temat powstało przez ostatnich dwadzieścia wieków mnóstwo prac autorstwa badaczy i myślicieli z bardzo różnych dziedzin - tak wiele, że próba choćby najbardziej pobieżnego ich omówienia musiałaby skończyć się fiaskiem. Jednakże niewiele jest wśród nich prac pisanych z perspektywy językoznawczej i traktujących metaforę jako problem przekładu » (2001: 90-91).

Gli interventi sulla metafora in chiave linguistica non prendono infatti quasi mai le dimensioni di un libro. Nella maggioranza dei casi sono degli articoli, il cui scopo è quello di presentare i procedimenti per la traduzione della metafora. Tra questi brevi interventi sulla metafora bisogna nominare l'articolo di Peter Newmark (1988), quello di Serena Ambroso e Alessandra Trecci (1999). Tra gli interventi piủ consistenti bisogna indicare il libro di Federica Scarpa (1989), che si basa sulla teoria di Newmark, e di Daniela Pirazini (1997).

${ }^{7}$ Per mancanza di spazio, le strategie di traduzione verranno presentate in generale senza eseguire paragoni tra strategie di traduzione attuate dalle traduttrici. Ogni strategia verra inoltre illustrata da esempi a scopo ulteriormente e strettamente esplicativo, cioè senza, ovviamente, pretendere di aver esaurito la problematica. 
Prima però di passare all'analisi, è necessario spiegare che cosa Lakoff e Johnson intendono per metafora in quanto, indubbiamente, non è facile discorrere della metafora senza averla definita. Negli occhi dei due studiosi, la metafora si mostra come una forma di pensiero che detiene il potere di strutturare la nostra percezione allo scopo di essere in grado di effettuare operazioni concettuali. Con questa osservazione Lakoff e Johnson fanno intendere che esistono delle metafore p.es. la discussione è una guerra, il tempo è denaro ecc. che riescono di cogliere, e di conseguenza riflettere in maniera verbale, d'altronde molto sintetica, il nostro modo di percepire la discussione e il tempo. Le metafore in questione ci aiutano a schedare nella lingua la nostra esperienza, in maniera tale che essa rimane impressa, o volendo, archiviata, nella sua semantica ${ }^{8}$.

Vediamo comunque da vicino di che cosa si tratti. Ognuno di noi ha sicuramente sentito più di una volta frasi come: Le tue richieste sono indifendibili. Egli ha attaccato ogni punto debole nella mia argomentazione. Se usi questa strategia, lui ti fa fuori in un minuto. Egli ha distrutto tutti i miei argomenti ${ }^{9}$. Tutti gli elementi in corsivo di queste frasi appartengono al campo semantico della guerra, in cui essi vengono applicati nel suo uso proprio. Tuttavia appena gli stessi elementi escono dal contesto guerresco, diventano di scatto metaforici. E questa dimensione figurata si manifesta grazie al fatto che tutte queste espressioni si basano su quello che Lakoff e Johnson chiamano il concetto metaforico ${ }^{10}$, vale a dire che un dato concetto viene modellato sulla base di un altro concetto. E così il concetto della discussione viene plasmato sul concetto della guerra, i quattro esempi precedenti sono infatti la realizzazione della metafora la discussione è una guerra. La stessa osservazione vale anche per la metafora il tempo è denaro, e per le sue realizzazioni: In questo risparmieremo alcune ore. Questa gomma a terra mi è costata un'ora. Ho

${ }^{8}$ Il meccanismo simile avviene, per esempio, anche nel caso delle espressioni fraseologiche. La fraseologia racchiude nella semantica di ogni lingua la mentalita degli utenti di questa lingua. A titolo d'esempio si potrebbe considerare la visione cattolica del mondo conservata nelle espressioni fraseologiche legata alla religione. La visione dell'angelo è da sempre associata alla dolcezza, la bontà e l'abilità. Per darne un esempio basta ricordare delle espressioni come: cantare, suonare, scrivere come un angelo (con impareggiabile bravura), mansueto (mite) come un agnello, buono come un angelo, qualcuno è un vero angelo (detto di persona buona, nobile, sempre pronta a portare aiuto a chi ne ha bisogno). Senza dimenticare tuttavia che accanto all'immagine positiva degli angeli vige una visione negativa del diavolo, chiamato spesso angelo caduto (infernale, delle tenebre) che rispecchia la veritá biblica che il diavolo è stato precipitato dall'arcangelo Michele nell'inferno. Il diavolo é spesso associato alla furbizia, diciamo: essere astuto (furbo) come il diavolo (piu del diavolo), avere il diavolo nell'ampolla (essere molto astuto e sagace), saperne una piu del diavolo (essere furbo, scaltro), sapere dove il diavolo tiene la coda (conoscere ogni tipo di inganno o malizia), ecc.

${ }^{9}$ I quattro esempi provengono da: Lakoff, Johnson (1998a: 22) e sono gli stessi che Lakoff e Johnson usano per presentare la metafora concettuale: la discussione è una guerra.

${ }^{10}$ Lungo tutto il libro di Lakoff e Johnson (1998a) sono infatti seminati diversi esempi dei concetti metaforici. Guardi, a titolo d'esempio, p. 30: le idee sono oggetti; p. 34: contento è su, triste è giü; p. 48: la mente è un macchina; p. 65: l'amore è un viaggio, ecc. 
sprecato un sacco di tempo per lei. Ho perso un sacco di tempo quando sono stato malato ${ }^{11}$.

I concetti che contribuiscono alla nascita dell'immagine metaforica vengono chiamati, nelle teoria cognitivista della metafora, il dominio di partenza e di arrivo. Jäkel chiarisce:

Metafory pojęciowe powstają jako skutek systematycznego powiązania dwóch różnych domen pojęciowych, z których jedna (X) funkcjonuje jako domena docelowa \{target domain), zaś druga (Y) jako domena źródtowa (source domain) metaforycznego rzutowania. Tym sposobem $\mathrm{X}$ rozumiana jest jako $\mathrm{Y}$, co czyni domenę pojęciową dostępną poznawczo, poprzez odwołanie się do innego obszaru doświadczenia [...] (Jäkel, 2003: 22).

Nel dominio di partenza si collocherebbero dunque la guerra e il denaro e nel dominio di arrivo la discussione e il tempo. La metafora invece non sarebbe altro che, in poche parole, il risultato dell'incrocio tra i due domini, che dà vita ad un nuovo dominio, esattamente come spiega Lorusso (2005: 14/15):

L'interprete della metafora, sovrapponendo (punto a punto) la struttura dell'uno sulla struttura dell'altro, è indotto, inferenzialmente, a creare un altro, nuovo, dominio concettuale che riproduce le caratteristiche salienti emerse dalla sovrapposizione. Questo movimento, da uno spazio mentale all'altro, verso un dominio del tutto nuovo, si produce a ogni occorrenza metaforica originale, perchẻ ogni metafora costruisce, localmente e temporaneamente, i suoi propri domini metaforici. Non c'è metafora che possa darsi entro un unico mental space. Se così fosse, altro non sarebbe che una sineddoche, $\mathrm{o}$ una metonimia.

Per quanto riguarda la natura del dominio di partenza e quello di arrivo, la teoria di Lakoff e Johnson sostiene che il dominio di partenza è di natura concreta in quanto conoscibile mediante l'esperienza reale mentre il dominio di arrivo è di natura astratta, fuori dalla portata dell'esperienza fisica. « Naczelna teza teorii głosi, że abstrakcyjne i kompleksowe domeny docelowe (X) są z reguły konceptualizowane przy pomocy bardziej konkretnych, prosto ustrukturyzowanych i poznawalnych zmysłowo domen źródłowych (Y) [...] » (Jäkel, 2003: 28) In rapporto, infatti, al metaforico passaggio dall'astratto al concreto, alla metafora è stata assegnata la funzione conoscitiva, la quale va di pari passo con la pratica di spiegare i termini astratti servendosi dei termini concreti, per il motivo che, come afferma Lakoff, «Metafora jest podstawowym mechanizmem, dzięki któremu rozumiemy abstrakcyjne pojęcia oraz dokonujemy abstrakcyjnego rozumowania. Większość treści, od najbardziej przyziemnych po najbardziej zawiłe teorie naukowe, można zrozumieć wyłącznie poprzez metaforę » (Jäkel, 2003: 34).

A questo punto rimane da chiarire soltanto il concetto di mapping, chiamato anche metaphorical projection, in italiano tradotto come mappatura, a volte anche proiezione. Per la spiegazione diamo la voce a Lakoff, che servendosi della metafora l'amore è un viaggio fornisce la definizione della mappatura (1998: 48-49):

${ }^{11}$ I quattro esempi provengono da: Lakoff, Johnson (1998a: 26) e sono gli stessi che Lakoff e Johnson usano per presentare la metafora concettuale: il tempo è denaro. 
In senso tecnico la metafora può essere definita come una mappatura (in senso matematico) da un dominio di partenza (nel nostro caso il viaggio) a un dominio di arrivo (nel nostro caso l'amore). La mappatura è strutturata con estrema precisione. E costituita infatti da corrispondenze ontologiche, attraverso le quali le entità nel dominio dell'amore (per esempio gli amanti, i loro scopi comuni, le loro difficoltà, la relazione amorosa) corrispondono sistematicamente alle entità del dominio del viaggio (i viaggiatori, il veicolo, i luoghi d'arrivo). [...] La mappatura L'AMORE È UN VIAGGIO è un gruppo di corrispondenze metaforiche che caratterizzano corrispondenze epistemiche attraverso la mappatua della conoscenza attorno al viaggio sulla conoscenza attorno all'amore usando la conoscenza che abbiamo acquisito e che usiamo per ragionare sul viaggio.

La mappatura nasce quindi sulla superficie concettuale tra due domini concettuali, cioè al livello della langue, in senso saussuriano del termine, e si manifesta sulla superficie linguistica in diverse espressioni metaforiche, ossia al livello della parole, sempre in chiave saussuriana. La mappatura si verifica per un motivo molto preciso, legato alla funzione conoscitiva della metafora in quanto il dominio di arrivo, più povero di quello di partenza, ha bisogno di essere arricchito da quest'ultimo.

Domena źródłowa jest znacznie precyzyjniej określona w naszym umyśle [...], ponieważ ma bogatszą strukturę i ta właśnie struktura jest nakładana (transponowana) na domenę docelową. Dlatego też rozumienie SPORU jako WOJNY wiąże się z nakładaniem bogatej struktury pojęcia <wojna> na prawdopodobnie ubogo ustrukturowane pojęcie <kłótnia> (Krzeszowski, 1998: 80).

3. Giudicando sulla base di come il traduttore decide di rendere il dominio di partenza, di arrivo e la mappatura della metafora nella lingua di arrivo, si possono evidenziare diverse strategie di traduzione, di cui ci occuperemo in seguito.

3.1. La meno impegnativa tra tutte le strategie applicate dalle tre traduttrici si effettua attraverso il mantenimento dello stesso dominio di partenza e di arrivo proposti nell'originale sulle pagine dell'opera tradotta, in pieno rispetto della mappatura inclusa nella metafora di partenza. In pratica la strategia in questione consiste nel rendere l'immagine metaforica ricorrendo alla traduzione parola per parola, cioè nel tradurre ogni componente linguistico della metafora sfruttando il suo esatto corrispondente presente nel lessico della lingua di arrivo, riportato su vari dizionari bilingui. Visto che questa strategia si basa sulla ricerca del corrispettivo letterale, può essere chiamata la traduzione letterale. In questo procedimento si distinguono due principali sottocategorie.

La prima abbraccia i casi della traduzione dei sintagmi di tipo nominale, verbale, avverbiale, ecc., trattandosi sia, in alcuni casi, di vere e proprie espressioni metaforiche perfettamente incorporate nel lessico delle due lingue, chiamate spesso morte e convenzionali, sia, in altri casi, di metafore del tutto nuove, chiamate anche inconvenzionali, originali. La traduzione consiste dunque nel ritrovare l'equivalente formale della metafora, cioè quello al livello del significante, dopo ovviamente essersi accertati che anche il significato corrisponde. A titolo illustrativo possono servire i seguenti esempi provenienti dal corpus: 
1. Guarda la strada, vede una piccola nube di polvere che si avvicina. (Castelli di rabbia, 1997: 126)

Patrzy na drogę, widzi zbliżający się mały obtok kurzu. (Zamki z piasku, 2006: 141)

La nube di polvere viene definita in Garzanti come « massa di particelle di varia natura sospese nell' aria », il che quadra pienamente con la traduzione polacca obłok kurzu. In questo caso il sintagma nominale, presente nella struttura della frase italiana, viene reso letterarmente dal suo corrispondente polacco. Anche nel caso dei sintagmi avverbiali o verbali il traduttore si limita a trovare l'equivalente formale senza che cambi il significato.

2. Nel prato, ai piedi della collina, c'e Elisabeth. Le hanno tolto tutti quei binari da davanti, le hanno giusto lasciato $i$ due che ha sotto le ruote. Se i treni naufragassero e le ferrovie fossero in cielo, sembrerebbe il relitto di un treno, posato sul fondale erboso del mondo. (Castelli di rabbia, 1997: 221)

Na łące u stóp wzgórza stoi Elisabeth. Usunięto sprzed niej wszystkie szyny, zostawiono tylko dwa kawałki pod samymi kołami. Gdyby pociąi tonęły, a linie kolejowe były na niebie, wyglądalaby jak wrak pociagu spoczywający na trawiastym dnie świata. (Zamki z piasku, 2006: 249)

3. Senza l'auspicato l'intervento della nostra scoperta, questo ornamento, tanto prezioso quanto delicato e simile per la grazia fragile delle sue forme segrete ad un fiore delicato che appassisce alla prima tempesta, resterebbe solo una fugace apparizione dello splendore, destinata, una volta trascorsa, a spegnersi sotto l'alito malefico della malattia, delle faticose esigenze dell'allattamento o della stretta funesta del busto crudele. (Castelli di rabbia, 1997: 139/140)

Bez pożądanej pomocy naszego wynalazku ornament ten, równie cenny, co delikatny i podobny w kruchym wdzięku swych sekretnych linii do delikatnego kwiatu więdnącego po pierwszej burzy, zachowałby jedynie cień byłego splendoru, którego przeznaczeniem, gdy czas jego minie. jest zgasną́ pod złowróżbnym tchnieniem choroby, męczących wymogów karmienia własną piersią i zgubnego uścisku okrutnego gorsetu. (Zamki z piasku, 2006: 156/157)

Sia nel caso della metafora sul fondale erboso del mondo che spegnersi sotto l'alito malefico della malattia viene applicata la traduzione parola per parola in quanto na trawiastym dnie świata e zgasnać pod ztowróżbnym tchnieniem choroby coincidono del tutto con le espressioni di partenza. Coincide anche lo status della metafora di partenza e di arrivo, nel primo esempio la metafora morta viene resa dalla metafora morta e nel secondo e nel terzo la metafora innovativa dalla metafora innovativa.

La seconda sottocategoria concerne la traduzione dei cosiddetti nomi propri metaforici. Da un punto di vista generale, si tratta semplicemente dei nomi propri utilizzati in qualità di metafore. Tuttavia, nel contesto metaforico gli stessi nomi propri perdono il loro carattere referenziale a favore della dimensione, si potrebbe 
dire, universale, vale a dire che essi non designano più un individuo oppure un oggetto, ma indicano una caratteristica oppure un insieme di caratteristiche proprie alla persona o all'oggetto evocati ${ }^{12}$. Vediamo gli esempi:

4. Erano i tempi in cui nella stazione di Buffalo c'erano tre orologi, ognuno con un'ora diversa, e sei ce n'erano nella stazione di Pittsburgh, uno per ogni linea ferroviaria che passava - era la Babele delle ore - e allora si capisce il rito della Londra - Dublino, treno postale - quell'orologio che va avanti e indietro, in una scatola di velluto, passando di mano in mano, prezioso come un segreto, prezioso come un gioiello... (Castelli di. rabbia, 1997: 58)

Były to czasy, gdy na stacji w Buffalo znajdowały sie trzy zegary, każdy wskazujący inną godzine, a sześć na stacji u Pittsburghu, po jednym dla każdej linii kolejowej, która tamtędy przebiegala - istna Wiéza Babel godzin - nietrudno więc zrozumieć rytuał pociagu pocztowego Londyn - Dublin - ten zegarek, który jeździ tak i z powrotem w aksamitnym pudełku, przechodząc $z$ ręki do ręki, cenny jak sekret, cenny jak klejnot... (Zamki z piasku, 2006: 62)

5. E nel finestrino - nel finestrino, di là dal vetro, sfilavano via i cocci di un mondo fatto a pezzi. perennemente in fuga, sminuzzato in migliaia di immagini lunghe un istante, strappato via da una forza invisibile. 'Prima che inventassero le ferrovie la natura non palpitava più: era una Bella Addormentata nel bosco' scrissero. (Castelli di rabbia, 1997: 61/62)

A w oknie - w oknie, po drugiej stronie szyby - przesuwły się odłamki świata w kawałkach, w wiecznej ucieczce, rozbitego na tysiące obrazów trwających każdy jedną chwilę, unoszonego niewidoczną siła. 'Przed wynalazkiem kolei natura przestała pulsować: była Śpiaca Królewna', napisano. (Zamki z piasku, 2006: 66/67)

Nel primo caso la Babele delle ore rimanda l'attenzione del lettore alla famosa Torre di Babele, chiamata in questo modo dal nome della città in cui gli uomini, secondo il racconto biblico, nella sua cieca presunzione di voler uguagliare Dio, avviaronò la costruzione di una torre altissima, che però non fu mai terminata perché avenne la confusione delle lingue, voluta da Dio come punizione, e non fu più possibile per $\mathrm{i}$ costruttori di comprendersi e in consegunza di collaborare. Partendo da questa ottica, la Babele delle ore viene ad indicare la confusione dei primi orari ferrovieri, diversi a seconda della stazione e della linea ferroviaria. Nel caso invece della Bella Addormentata nel bosco viene evocata l'immagine della principessa del mondo delle fiabe, risvegliata dal sonno profondo e lungo, con lo

12 Jonasson spiega il meccanismo in questione con questi termini: "Car le Npr [nom propre métaphorique] a maintenant une fonction descriptive [...] et non plus une fonction identifiante, et les propriétés retenues sont donc tout autres que celles retenues dans son emploi référentiel. C'est maintenant une ou plusieurs propriété(s) caractéristiques(s) ou un destin particulier qui comptent. Ces aspects permettent d'êtablir un modèle mental du référent original qui en est considéré comme l'incarnation ou le paragon. Ce modèle est le membre central idéal d'une catégorie prototypique dont tous les membres ont une ressemblance plus ou moins parfaite avec le membre modele " (Jonasson. 1994: 220). 
scopo di indicare il fatto che anche la natura, prima della rivoluzione ferroviaria, sembrava essere statica e dormente, e invece vista dal finestrino del treno in movimento sembra galoppante. Anche in questi casi, il traduttore, adoperando la resa letterale delle due metafore, ha rispettato la loro natura (nomi propri metaforici) e il significato racchiuso nell' originale.

3.2. Va comunque subito chiarito, altrimenti si corre il rischio di indurre in errore facendo credere che la traduzione automatica è tutt'altro che un sogno mai avverato, suscitato dalle ricerche iniziate intorno agli anni Quaranta ${ }^{13}$, che l'attuarsi del processo sopradescritto non sempre basta per trasferire un 'immagine metaforica da una lingua all'altra. Il traduttore viene infatti molto spesso messo in condizione di dover far fronte a diversi problemi, non sempre facili da risolvere, e non sempre ne sa uscire vincitore. Il traduttore si vede a volte costretto a dover ricorrere alla cosiddetta demetaforizzazione, in altre parole, alla perdita della dimensione metaforica. Nell'applicazione di questo procedimento traslativo non solo non avviene quello che Lakoff e Johnson chiamano la mappatura dal dominio di partenza al dominio di arrivo, ma addirittura non c'è nessun dominio di partenza in quanto avviene la sua soppressione a favore del dominio di arrivo che allarga la sua estensione. Il traduttore rimane infatti nel dominio di arrivo dell'immagine metaforica ed è là che cerca di realizzare la trasposizione della metafora. Il procedimento più ricorrente di questa strategia è la riformulazione che consiste appunto nel parafrasare il contenuto dell'immagine metaforica tramite la forma di dimensioni ridotte (a volte si tratterà semplicemente di sostituire un sostantivo, un verbo o un aggettivo utilizzati al senso metaforico da un sostantivo, un verbo o un aggettivo privi di questa dimensione) oppure sviluppate, fino ad arrivare a vere e proprie spiegazione del significato metaforico.

Senza dubbio gli esempi seguenti rappresentano i casi della riformulazione, si potrebbe dire, breve, abbastanza sintetica in quanto un elemento solo viene sostituito da un altro elemento singolare, così assalita da palpitazioni, vertigini, sudori freddi e stordimenti viene sostituito da dostawała palpitacji, zawrotów gtowy, zimnych potów i oszotomienia, la magra consolazione da jedyne pocieszenie, oppure tutta l'espressione viene accorciata come nel caso quando cadeva la luce da przed wieczorem:

6. La signora Gina Biglia, la mamma di Graziano, soffriva dì ipertensione. Di minima aveva centoventi e di massima oltre centottanta. Le bastava un' agitazione, un'emozione e subito veniva assalita da palpitazioni, vertigini, sudori freddi e stordimenti. (Ti prendo e $t i$ porto via, 1999: 54)

Pani Gina Biglia, mama Graziana, cierpiała na nadciśnienie. Miała ponad sto osiemdziesiąt na sto dwadzieścia. Wystarczało lekkie zdenerwowanie albo wzruszenie i natychmiast dostawata palpitacji, zawrotów głowy, zimnych potów i oszotomienia. (Zabiorę cię ze soba, 2002: 50)

${ }^{13}$ Cfr. Nergaard (1993: 11). 
7. Sarebbe tornata al villaggio e avrebbe rivisto i suoi tre figli, questa era la magra consolazione. (Ti prendo e ti porto via, 1999: 334)

Wróci do wioski i zobaczy swoich trzech synów, to było jedyne pocieszenie. (Zabiorę cię ze sobq, 2002: 312)

8. Il signor Rail andava ogni giorno a trovarlo, quando cadeva la luce. (Castelli di rabbia, 1997: 132)

Pan Rail odwiedzał go codziennie przed wieczorem. (Zamki z piasku, 2006: 148)

Tuttavia la situazione contraria si presenta nel caso degli esempi successivi in cui il traduttore rendere le metafore servendosi delle spiegazioni, sostituendo infatti un filo di voce da ledwo dostyszalnym gtosem, anche lui agli sgoccioli da on też miat już zamiar z tym skończyć, e Le secca da Nie ma pan nic przeciwko temu:

9. E allora Jun si voltò lentamente e rientrǒ in casa. Scivolò nel buio delle stanze e sparì. Non si voltǒ, il signor Rail, se ne rimase a fissare Elisabeth, laggiu, e solo dopo qualche istante disse qualcosa, ma molto piano, come a se stesso, in un filo di voce. (Castelli di rabbia, 1997: 113)

I wtedy Jun odwróciła się powoli i weszła do domu. Wsunęła się w mrok pokojów i znikła. Pan Rail nie odwrócił się, nadal patrzył w dół, na Elisabeth, i dopiero po kilku chwilach powiedział coś, ale bardzo cicho, jakby do siebie, ledwo dostyszalnym glosem. (Zamki z piasku, 2006: 126)

10. L'ultimo incontro lo fece contro un nero di Filadelfia, anche lui agli sgoccioli. (City, 1999: 71)

Ostami pojedynek stoczył z pewnym Murzynem z Filadelfii, on też miat już zamiar z tym skończyć. (City, 2000: 82)

11. - Le secca se finisco la telefonata? (City, 1999: 17)

- Nie ma pan nic przeciwko temu, żebym skończyła rozmowę? (City, 2000: 16)

A volte però la riformulazione comporta degli ulteriori cambiamenti nella dimensione diafasica della lingua, determinando il variare dei registri espressivi, di solito dal familiare al neutrale, come negli esempi 11 e 12:

12. Ma Mimmo non aveva alcuna intenzione di seguire le avventure del capitano Kirk e compagni. Accadeva di rado che sua madre si schiodasse da casa e ne voleva approfittare. Stringeva e palpava la fidanzata come un polipo in amore. (Ti prendo e ti porto via, 1999: 218)

Tyle, że Mimmo nie miał najmniejszego zamiaru śledzić przygód kapitana Kirka i jego towarzyszy. Nieobecność matki była rzadką okazją i chciał ją wykorzystać. Ściskał i obmacywał narzeczoną niczym zakochany polip. (Zabiorę cię ze soba, 2002: 203)

$\mathrm{Al}$ fine di completare la presentazione, bisogna accennare alla situazione in cui il traduttore adopera la tecnica di demetaforizzazione benché esistano corrispettivi 
letterali per l'immagine metaforica, è il caso, per esempio, del verbo nascere, scoppiare, dell'espressioni nel cuore di, il cuore di, ecc.

16. Poi gli nacque un dubbio legittimo. (Fango, 1996: 23)

Potem jednak pojawiła się pewna uzasadniona wątpliwość. (Błoto, 2001: 22)

17. La prima cosa è il mio nome, la seconda quegli occhi, la terza un pensiero, la quarta la notte che viene, la quinta quei corpi straziati, la sesta è fame e la settima è orrore, l'orrore, che scoppia di notte - di nuovo la notte - l'orrore, là ferocia, il sangue, la morte, l'odio, fetido orrore. (Oceano mare, 1993: 104)

Po pierwsze moje nazwisko, po drugie tamte oczy, po trzecie jedna myśl, po czwarte noc, która zapada, po piąte te udręczone ciała, po szóste głód i po siódme strach, strach pojawiajacy się noca - znowu noc - strach, okrucieństwo, krew, śmierć, nienawiść, nikczemny strach. (Ocean morze, 2001: 122/123)

18. Il signor Rail e Hector Horeau, uno seduto di fronte all'altro, nel cuore dell'inverno, nel cuore della grande casa: silenziosa. (Castelli di rabbia, 1997: 195)

Pan Raił i Hector Horeau siedzą naprzeciw siebie, w samym środku zimy, w samym środku wielkiego domu: pogrążonego w ciszy. (Zamki z piasku, 2006: 218)

Tuttavia, a prescindere da 16, 17 e 18, anche gli esempi 6, 7, 8, 9 e 10 possono essere tradotti, ricorrendo, questa volta, alla traduzione letteraria, di cui nel 3.4. In questa ottica gli equivalenti polacchi sarebbero: (6) padała ofiarq palpitacji, zawrotów gtowy, zimnych potów i oszołomienia, (7) marne to byto pocieszenie, (8) gdy zapadat zmrok, (9) stabym głosem, pod nosem, resztkami gtosu, sttumionym głosem, (10) on też na finiszu. La situazione pare invece più complessa nel caso di 11 e 12, perché, anche se le traduzioni come (11) wkurzy to pana jeśli dokończe rozmowe e (12) Rzadko się zdarzato, żeby matka ruszata się z chaty i chciat z tego skorzystać rispettano il registro dell'originale, fanno smarrire la dimensione metaforica.

3.3. Tuttavia, la diminuzione dell'effetto delle metafore di partenza sul testo di arrivo non si realizza esclusivamente attraverso lo scioglimento della dimensione metaforica pur lasciando inalterato il significato. A volte il traduttore non solo non mantiene la metafora, ma decide pure di cancellare qualsiasi incidenza sul significato di quest'ultima, facendo uso dell'abolizione. Questa strategia potrebbe venire definita come la più categorica di tutte in quanto si basa sul meccanismo di soppressione assoluta tanto del dominio di partenza quanto addirittura di quello di arrivo, di conseguenza viene eliminata anche la mappatura. Questo procedimento porta dunque alla cancellazione di ogni componente dell'immagine metaforica, dimodoché non se ne manifesti nessuna traccia.

19. Sì, dormiva troppo. Ma la sera tornava a casa distrutta senza nessuna voglia di uscire, di vedere nessuno. Come si fa a uscire, a motivarsi quando le palpebre ti pesano come due ghigliottine? (Fango, 1996: 155) 
Tak, spała zbyt wiele. Ale kiedy wracała wieczorami do domu, nie miała już najmniejszej ochoty wyjść, spotkać kogokolwiek. Jak można prowadzić aktywne życie towarzyskie, kiedy powieki ciążą ci niczym dwie gilotyny? (Błoto, 2001: 160)

20. Il pastore disse che temeva di no. Allora si aprì un dibattito, e tutti provarono a scavare nella memoria per riuscire a ricordarsi una cosa buona, anche una sola, che Bird avesse fatto nella vita. (City, 1999: 300)

Pastor odparł, iż obawia się, że nie. Wtedy rozpętała się dyskusja, wszyscy próbowali coś sobie przypomnieć, jakiś dobry uczynek, choćby jeden, jaki można by przypisać Birdowi, zabawne, ale przychodziły im do głowy same draństwa. (City, 2000: 376)

21. Disse anche che nell'armadio della camera da letto c'erano ancora tutti i suoi vestiti, e che lei sapeva imitare le voci dei cantanti, cantava con la voce di Marilyn Monroe che sembrava sputata lei. (City, 1999: 127)

Dodał, że w szafie, w sypialni, wiszą wszystkie jej ubrania i że ona umiała naśladować glos piosenkarek, śpiewała głosem identycznym jak Marilyn Monroe. (City, 2000: 154)

22. La solita nuvola di sabbia e tramonto, come ogni sera, fumata dal vento sulla terra e dentro il cielo, mentre Melissa Dolphin spazza la strada davanti a casa, frullata dal fiume d'aria rotondo. con irragionevole cura, e inutile, spazza. Ma portando i suoi anni, sessantatré, con calma e gratitudine. (City, 1999: 50)

Ta sama, jak co wieczór, chmura kurzu i zachód słońca, chmura wzbita przez wiatr na ziemi i w niebie, a tymczasem Melissa Dolphin zamiata ulicę przed domem, $\mathrm{z}$ irracjonalną i daremną starannością. Dźwiga ciężar swoich sześćdziesięciu trzech lat ze spokojem i wdzięcznością. (City, 2000: 55)

A volte succede che all'appello manca addirittura tutto il frammento che conteneva l'immagine metaforica:

23. L'aveva detto con una voce che sembrava nuova. sembrava che gliel'avessero restituita in quel momento, lavata e stirata. Come se l'avesse mandata in tintoria. (City, 1999: 248)

Tutto il frammento manca nella traduzione in polacco.

3.4. A questo punto sporge spontanea una domanda molto semplice e allo stesso momento molto concreta: si può tradurre la metafora al di là degli esempi 1 , $2,3,4,5,16,17,18$ per cui sembra valere l'autonomia del significato postulata dalla linguistica strutturalista e generativista? A questa domanda è molto difficile rispondere con un sì o con un no categorico, dato che la questione della traduzione è ben lontana dalle certezze delle scienze matematiche. Nella nostra opinione, un tentativo di risposta a questa domanda potrebbe costituire l'ultima strategia di traduzione riscontrata nel materiale analizzato. Questo modo di tradurre, chiamato la traduzione non letterale, in cui il traduttore cerca di rendere l'immagine metaforica facendo uso di un'altra immagine sempre di valore metaforico, si realizza attraverso due fondamentali modi di procedere individuati in base a come il traduttore tratta il dominio di partenza. 
Nel caso in cui il dominio di partenza rimane uguale scatta il meccanismo della selezione, cioè il traduttore cerca di scegliere il sinonimo ${ }^{14}$ più adatto per il termine usato nell'originale dando contemporaneamente nascita ad una rete diversa di connotazioni.

24. Hervé Joncour era all'estremo opposto della stanza: era assediato dal profumo dolciastro delle donne che gli stavano attorno e sorrideva imbarazzato agli uomini che si divertivano a raccontargli storie che lui non poteva capire. (Seta, 1996: 54)

Hervé Joncour był po drugiej stronie pokoju: osaczony słodkawym zapachem otaczajacych go kobiet, uśmiechał się z zakłopotaniem do mężczyzn zabawiających go historyjkami, ktŏrych nie mógł zrozumieć. (Jedwab, 1998: 58)

Il verbo assediare fa pensare a delle azioni belliche il cui scopo è quello di isolare il nemico, per poi sconfiggerlo più facilmente, il sinonimo che Garzanti mette in prima posizione per il verbo assediare è accerchiare che in polacco vuol dire appunto osaczyć, anche in polacco i significati di oblegać e di osaczyć sono sinonimici e rimandano al campo semantico delle operazioni belliche. Le stesse osservazioni valgono per gli esempi successivi.

25. - E i naufragi? Le tempeste, i tifoni, tutte quelle cose lì... Perché mai dovrebbe ingoiarsi quelle navi, se sono i suoi occhi? (Oceano mare, 1993: 81)

- A morskie katastrofy? Burze, tajfuny, te wszystkie rzeczy... Dlaczego niby miałoby pochtaniać okręty, skoro są jego oczami? (Ocean morze, 2001: 93)

Il verbo ingoiare fa venire in mente un'immagine di qualcuno che inghiotte, per lo più avidamente $\mathrm{e}$ in fretta, anche il verbo pochtaniać viene definito « zjeść, wypić coś łapczywie, w dużych ilościach » e costituisce il sinonimo per potykać.

Per quanto riguarda invece la situazione in cui il dominio di partenza cambia, la maggioranza dei casi riscontrati, la trasformazione può avvenire per vari motivi, anche se, a volte, potrebbe essere evitata perché sarebbe possibile la traduzione letterale, più fedele all'originale. Vediamo gli esempi:

26. Ronzano mille cose, nella testa di Bartleboom, mentre ritrae le mani, tenendole aperte, come se a chiuderle scappasse tutto. (Oceano mare, 1993: 91)

Tysiące myśli kottuje się w głowie Bartlebooma, gdy cofa dłonie, otwarte, jakby po zamknięciu ich wszystko miało zniknąć. (Ocean morze, 2001: 106)

27. Tecnicamente parlando, Gould era un genio. A stabilirlo era stata una commissione di cinque professori che l'aveva esaminato, all'età di sei anni, sottoponendolo a tre giorni di test. In base ai parametri Stocken, risultǒ appartenere alla fascia delta: a quei livelli l'intelligenza è una macchina ipertrofica di cui è difficile intuire i limiti. Provvisoriamente gli assegnarono un QI di 108, cifra abbastanza mostruosa. (City, 1999: 31)

${ }^{14}$ Nel caso 25 e 26 il sinonimo si impone spontaneamente in quanto le espressioni pochtaniać okręty rivolta ai fenomeni marittimi e myśli kotłujq się/kłębiq się sono locuzioni quasi lessicalizzate nella lingua polacca. 
$\mathrm{Z}$ naukowego punktu widzenia Gould był geniuszem. Ustaliła to komisja złożona z pięciu profesorów, która przeegzaminowała go, poddając trzydniowym testom, kiedy miał sześć lat. Na podstawie parametrów Stockena okazało się, że mieści się w przedziale delta: na tym poziomie inteligencja stanowi hipertroficzną machinę, której granice trudno nawet sobie wyobrazić. Tymczasem przyznali mu QI 108, cyfrę dość kolosalnq. (City, 2000: 31)

28. Solo la scienza può certe cose, questa è la verità. Spazzare secoli di schifo - l'orrendo mare grembo di corruzione e morte - e inventare quell'idillio che a poco a poco si diffonde su tutte le spiagge del mondo. (Oceano mare, 1993: 46)

Tylko nauka może dokonać pewnych rzeczy, oto cała prawda. Odrzucić wieki obrzydzenia - ohydy morza, kolebki zniszczenia i śmierci - i wymyślić tę idyllę, która powoli rozprzestrzenia się na wszystkie plaże świata. (Ocean morze, 2001: 50/51)

Nel primo il verbo ronzare viene reso nella traduzione tramite il verbo kottować się, che non rimanda alla stessa immagine creata da ronzare, il quale fa pensare a insetti come api, vespe, mosche che emettono, definisce Garzanti, un rumore vibrante, sordo e prolungato, in conseguenza del loro volare soprattutto in cerchio. Kottować się, invece, richiama all'attenzione un kociot in cui si mescolano senza tregua vari liquidi, per cui kottować się significa « być w ciagłym, bezładnym ruchu, gwałtownie się poruszać; kłębić się, burzyć się, wirować, wrzeć, bulgotać », invece ronzare vuol dire girare intorno ad un luogo, un oggetto, una persona. La situazione simile si è creata anche nel secondo esempio, dove l'aggettivo mostruoso viene tradotto da kolosalny, il primo fa pensare ad un mostro, il secondo a kolos, con il significato comune di qualcosa, in questo caso di una cifra, straordinariamente grande. Infine nel terzo esempio figurano spazzare e odrzucić che significano sbarazzarsi di qualcosa in modo da poterlo dimenticare, con la precisione che i domini di partenza sono diversi, nel caso di spazzare i secoli in questione devono essere appunto eliminati servendosi di una scopa, e nel caso di odrzucić i secoli vengono mandati lontano indirizzati in una data direzione con l'aiuto di un'energia necessaria.

Dalla questione di sostituzione di un dominio di partenza da un altro, non può che derivare un altro problema, quello del cambiamento dello status della metafora, che da innovativa diventa morta, come nell'esempio seguente dove gli rimase appiccicato in testa viene tradotto da wbita mu się do gtowy (anche: dlatego też ta nazwa wbiła mu się w pamięć oppure zapisała mu się w pamięci, wyryła mu się w pamięci)

29. È lì che Tool lesse quel nome: Quinnipak. Per uno che sta andando in galera, vedere una freccia che porta altrove dev'essere come guardare in faccia l'infinito. Qualsiasi cosa ci fosse, laggiủ, era comunque vita, e non galera. Così quel nome gli rimase appiccicato in testa. (Castelli di rabbia, 1997: 242)

To na niej Tool przeczytał tę nazwę: Quinnipak. Dla kogoś, kto jedzie do więzienia, zobaczyć strzałkę prowadzacą gdzie indziej to tak, jakby spojrzeć w twarz nieskoń- 
czoności. Cokolwiek by tam było, musiało to być życie, nie więzienie. Dlatego ta nazwa wbiła mu się do glowy. (Zamki z piasku, 2006: 270/271)

4. Concludendo possiamo constatare quanto segue:

- Il traduttore, posto di fronte al compito di trasferire la metafora da una lingua all'altra, applica, con diverso successo, in linea di massima, quattro principali strategie: i) la traduzione letterale che è ridotta allo sforzo di trovare una corrispondenza tra parole appartenenti a due lingue diverse, il che è giustificato dal fatto che in questi casi l'identità formale al livello del contenuto implica l'identità al livello dell'espressione, la traduzione equivale dunque alla transcodificazione, il ruolo del traduttore è piuttosto limitato in quanto quest'ultimo deve attingere solamente alle sue conoscenze linguistiche, per quanto riguarda la traduzione dei sintagmi, soprattutto quelli lessicalizzati, e, per quanto concerne la traduzione dei nomi propri metaforici, ricorrere al suo sapere culturale; ii) la demetaforizzazione che provoca una sorta di infedeltà nei confronti del testo originale attraverso l'abbandono del carattere metaforico del testo di partenza, il che costituisce dalla parte del traduttore una grave mancanza $(6,7,8,9,10)$ e una spensieratezza nei casi in cui è possibile trovare un equivalente soddisfacente, ricorrendo semplicemente alla traduzione letterale (esempi 16, 17, 18), Pirazzini (1997: 75) giudica questa strategia in seguenti termini: «Sostituire, per esempio, il sogno della luna con il senso che esso trasmette significa produrre una parafrasi interminabile più lunga dell'enunciato, la quale risulterà, sempre e comunque, fondamentalmente goffa. Traducendo così, si perde il maggior grado di informatività della metafora rispetto ad una possibile riformulazione letterale »; iii) l'abolizione della metafora, che, nella nostra opinione, è un errore molto grave, dato che, applicando questa strategia, il traduttore non sopprime soltanto la metafora, ma nell'atto di non tradurre elimina contemporaneamente tutta la ricchezza semantica che la metafora porta con sé. A pagarne le conseguenze non è dunque solo la stilistica, ma anche la semantica, perché cambia il valore informativo del testo di arrivo. Questa negligenza è ancora più grave se si prende in considerazione che in diversi casi, la traduzione sarebbe possibile, p.es. negli esempi distrutta (19), scavare nella memoria (20) oppure sputata lei (21) si può ricorrere alla traduzione letteraria: wypompowana, wykończona; sięgnać do pamięci, szukać w pamięci, wysilać pamięć oppure wykapana ona, ma, tant'è vero che già il frammento frullata dal fiume d'aria rotondo (22) pone dei problemi, tutt'altro che banali; iv) la traduzione letteraria che esige. dalla parte del traduttore, la capacità di sapere utilizzare la parola, d'essere inventivo al livello lessicale, e ovviamente di saper leggere il contenuto del testo originale, d'essere cioè vigile al livello interpretativo, il che vuol dire che il traduttore deve prima di tutto impegnarsi a comprendere la specificità del messaggio contenuto nella lingua di partenza e in seguito mettere in gioco tutte le risorse della lingua d'arrivo al fine di ottenere lo stesso effetto stilistico e semantico nella traduzione;

- Le strategie traslative presentate mettono in evidenza due tendenze, molto distinte bensì opposte; la prima è caratterizzata da un grande sforzo, compiuto dal 
traduttore al fine di rendere le metafore mantenendo il loro dominio di partenza originale (la traduzione letterale) oppure modificandolo leggermente e perfino cambiandolo (il caso della traduzione letteraria); la seconda invece consiste nel voler in qualche maniera disfarsi della metafora concepita come elemento difficile da tradurre (la demetaforizzazione e l'abolizione della metafora);

- Le difficoltà che il traduttore incontra nel processo traslativo delle metafore dipendono da quattro fattori, che nella maggioranza dei casi si sovrappongono, il che significa che la traduzione riposa nella stessa maniera su di essi, essa costituisce dunque una sorta di risultante di questi quattro vettori: i) la specificità della lingua di partenza e di arrivo, soprattutto le risorse lessicali e sintattiche, a volte incompatibili; ii) la categorizzazione del mondo per il fatto che ogni lingua ha i suoi modi di concettualizzare la realtà, a volte molto lontani; iii) la traduzione deve tendere a mantenere lo stile proprio dell'autore del testo tradotto, e in conseguenza, se questo stile è altamente metaforico, con tante metafore innovatrici, il traduttore deve sapere rendere questo tratto caratteristico; iv) anche le competenze del traduttore lasciano il segno sulla traduzione, il traduttore in quanto intermediario deve conoscere la particolarità della lingua di partenza e d'arrivo sotto i tre punti di vista elencati sopra.

Alla luce di quanto detto sopra bisogna porsi la domanda se la tendenza ad evitare la metafora potrebbe essere in qualche maniera giustificata per esempio dal fatto che la lingua di arrivo ha delle risorse lessicali diverse dalla lingua di partenza oppure per il motivo che le due lingue funzionano in contesti culturali diversi, e in una cultura una data metafora può essere accettata senza problemi, in un'altra invece in maniera minore o per niente, in quanto può sembrare estremamente strana.

Non senza importanza è ovviamente la questione, se alle suddette domande diamo una risposta affermativa, dell'impoverimento del contenuto del testo originale rispetto alla traduzione e del mancato equilibrio di elementi che costituiscono lo stile specifico dell'autore.

C'è chi sostiene che tali insufficienze sono giustificabili dalla strategia di traduzione chiamata la compensazione, che consiste nel creare la metafora là dove l'originale non la usa al fine appunto di compensare gli squilibri stilistici. Dello stesso parere non è però Pirazzini (1997: 200) che constata:

La differenza cardinale è che l'uso figurato, nella maggior parte dei casi, trasmette una valutazione sul referente o sul concetto che esso designa. In altri termini, non è la stessa cosa descrivere una persona come: una belva o come: molto cattiva.

L'aggiunta di significato metaforico nella traduzione, quindi, porta ad una quantità di informazioni che, in linea di massima, non è mai la stessa di quella di partenza.

A questa osservazione va aggiunta anche la preoccupazione che la compensazione può implicare il rischio, se non addirittura la tentazione, di farla diventare una 
via di fuga di fronte alle metafore difficili da tradurre, allo scopo di liberare il traduttore dalla necessità di sforzarsi nella ricerca di altre soluzioni.

Forse la toccasana a tutti i dubbi sorti riguardo alla traduzione delle metafore sarebbe custodita nella seguente riflessione espressa da Cicerone: « In essi io non ho creduto di rendere parola con parola, ma ho mantenuto ogni carattere e ogni efficacia espressiva delle parole stesse. Perché non ho pensato più conveniente per il lettore dargli, soldo su soldo, una parola dopo l'altra: piuttosto, sdebitarmene in solido » (1993: 58) Vale dunque sempre e comunque la regola d'oro, da rispettare in ogni traduzione, cioè quella di tradurre a senso, cercando di salvare la dimensione metaforica ricorrendo alla resa letterale e quella letteraria.

\section{BIBLIOGRAFIA}

Ambroso S., Trecci A. (1999), « La traduzione della metafora », in: P. Pierini, L'atto del tradurre. Aspetti teorici e pratici della traduzione, Roma : Bulzoni Editore.

Cicerone M.T. (1993), "Qual è il miglior oratore », in: Nergaard S. (a cura di), La teoria della traduzione nella storia (trad. di G. Tissoni), Milano : Bompiani.

Dirven R., Verspoor M. (a cura di), (1999), Introduzione alla linguistica: un approccio cognitivo, Bologna : Clueb.

Gaeta L., Nuraghi S. (2003), Introduzione alla linguistica cognitiva, Roma : Carocci.

Jäkel O. (2003), Metafora $w$ abstrakcyjnych domenach dyskursu: kognitywno-lingwistyczna analiza metaforycznych modeli aktywności umystowej, gospodarki i nauki (trad. di M. Banaś, B. Drag), Kraków : Universitas.

Jonasson K. (1994), Le nom propre. Constructions et interprẻtations, Paris : A. Michel.

Krzeszowski T. (2005), « Aksjologiczne aspekty metafor », in: W. Kubiński, R. Kalisz, E. Modrzejewska, Językoznawstwo kognitywne. Wybór tekstów, Gdańsk : Wydawnictwo Uniwersytetu Gdańskiego.

Lakoff G. (1991), «Una figura del pensiero », in: C. Cacciari, Teorie della metafora. L'acquisizione, la comprensione, e l'uso del linguaggio figurato, Milano: Cortina Editore.

Lakoff G., Johnson M. (1998a), Metafora e vita quotidiana (trad. di P. Violi), Milano : Bompiani.

Lakoff G., Johnson M. (1998b), Elementi di linguistica cognitiva (trad. di M. Casonato, M. Cervi), Urbino : Quattro Venti.

Lorusso A.M. (2005), Metafora e conoscenza: da Aristotele al cognitivismo, Milano : Bompiani.

Łagoda K. (2006), « Niccolò Ammaniti. Nobilitacja kanibala », in: H. Serkowska, Literatura wtoska $w$ toku, Wrocław : Ossolineum.

Manetti G. (2005), «Aristotele e la metafora. Conoscenza, similarità, azione, enunciazione », in: A.M. Lorusso, Metafora e conoscenza: da Aristotele al cognitivismo, Milano : Bompiani.

Nergaard S. (a cura di), (1993), La teoria della traduzione nella storia, Bompiani : Milano.

Newmark P. (1988), La traduzione: problemi e metodi (trad. di F. Flangini), Milano : Garzanti.

Pirazzini D. (1997), Cinque miti della metafora nella Übersetzungswissenschaft. Problemi di traduzione delle immagini figurate nella coppia di lingue: Tedesco (Lingua di Partenza) - Italiano (Lingua d'arrivo), Peter Lang : Europäischer Verlag.

Rollo A. (2004), La linguistica cognitiva: dalle teorie alla grammatica, Lecce : Adriatica editrice salentina. 
Scarpa F. (1989), La traduzione della metafora, Roma : Bulzoni.

Stockwell P. (2006), Poetyka kognitywna (trad. di A. Skucińska), Kraków : Universitas.

Świątek J. (1998), W świecie powszechnej metafory, Kraków : PAN.

Tabakowska E. (2001), Językoznawstwo kognitywne a poetyka przektadu (trad. di A. Pokojska), Kraków : Universitas.

Traini S. (2001) La connotazione, Milano : Bompiani.

Ugniewska J. (2006), « Alessandro Baricco, czyli jak uwieść czytelnika », in: H. Serkowska, Literatura wtoska w toku, Wrocław : Ossolineum.

\section{TESTI SPOGLIATI}

Ammaniti N. (1996), Fango, Milano : Mondatori.

Ammaniti N. (1999), Ti prendo e ti porto via, Milano : Mondatori.

Baricco A. (1993), Oceano mare, Milano : Rizzoli.

Baricco A. (1996), Seta, Milano : Rizzoli.

Baricco A. (1997), Castelli di rabbia, Milano : Rizzoli.

Baricco A. (1999), City, Milano : Rizzoli.

\section{EDIZIONI POLACCHE}

Błoto, trad. di D. Duszyńska, Muza, Warszawa 2001.

Zabiorę cię ze soba, trad. di D. Duszyńska, Muza, Warszawa 2002.

Ocean morze, trad. di H. Kralowa, Czytelnik, Warszawa 2001.

Jedwab, trad. di H. Kralowa, Czytelnik, Warszawa 1998.

Zamki z piasku, trad. di H. Kralowa, Czytelnik, Warszawa 2006.

City, trad. di J. Ugniewska, Czytelnik, Warszawa 2000.

\section{DIZIONARI}

Garzanti in versione elettronica

Stownik jęzka polskiego PWN in versione elettronica 